\title{
Design and Implementation of Modern Agriculture Monitoring System
}

\author{
Dan Li \\ College of Electrical and Information Engineering, Jilin Agricultural Science and Technology \\ University, Jilin City, China \\ 653301981@qq.com
}

Keywords: ARM microprocessor; Temperature sensor; TCP/IP protocol; Intelligent greenhouse

\begin{abstract}
With the rapid development of modern agriculture, the timeliness and accuracy of monitoring agricultural environment factors is also required. This paper studies the application of ARM microprocessor in modern agricultural intelligent greenhouse monitoring field. It designs that several high precision digital temperature sensors (AD7416) can simultaneously monitor many different position greenhouse temperature; it adopts that ARM microprocessor provides 3 level instruction pipelines; it designs the communication interface to portable computer and network communication method of TCP/IP protocol. The system has high monitoring accuracy, low cost, and other advantages.
\end{abstract}

\section{Introduction}

In modern agriculture, we not only take advantages of natural resources, but also environmental factors to get the best condition of crop growth ,such as temperature, humidity and light which may be controlled by intelligent greenhouse environment monitoring system. The best condition can increase crop yield, improve quality, regulate the growth period, and improve the economic efficiency. It is very important to monitor environmental factors timely and accurately. The modern intelligent greenhouse is the prevalent development of greenhouse, because of its energy saving, environmental protection and improving the working conditions. Therefore, it is very meaningful and important that this paper deals with the hardware structure and software algorithm problems of intelligent greenhouse environment monitoring system. [1]

In order to monitor the greenhouse temperature accurately and in real time, and in order to research greenhouse temperature effects on agricultural production more easily, this paper uses the technology of single-chip microcomputer and digital sensor technology, to design and develop intelligent greenhouse environment monitoring system based on S3C44B0X microprocessor. [2] This system takes the high speed and low power ARM S3C44B0X microprocessor as control core, high precision digital temperature sensor as front-end acquisition module. The system carries out the functions of measuring, displaying, storing, and transmitting the multiple position greenhouse temperature. It is easy to operate, high monitoring accuracy, and low cost system.

\section{High Level Design of Hardware Platform}

One machine, which is a common temperature instrument in many markets, can only measure one location temperature at the same time. In order to show the results are universal, it is common to frequently change the position measured by the machine. [3] To solve the problem, this paper makes only one greenhouse temperature monitoring system with many temperature detection headers. High precision digital temperature sensor of each detection header can communicate with the central control unit through the serial bus.

The temperature argument value, which measured by the greenhouse temperature monitoring system, can be displayed on the LED Seven-Segment digital tubes in real-time, or can be stored in the system's internal part, also can be directly obtained through the serial interface to portable computer, for more complex data analysis operations and data printing. High level hardware platform design as shown in Fig. 1, 


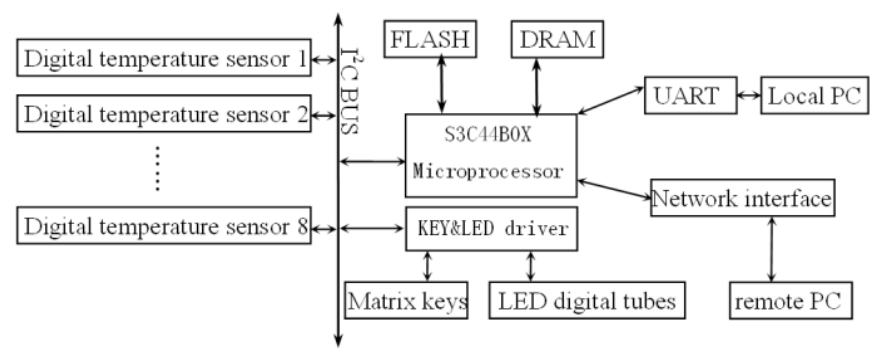

Figure 1. High level hardware platform diagram

\section{Design of Central Control Module}

In order to improve data processing capability of greenhouse temperature monitoring system, and to upgrade its performance to the high-end temperature measurement instrument, this paper uses Samsung's high performance-to-price ratio and high performance 16/32 bit RISC microprocessor S3C44B0X chips. S3C44B0X uses ARM7TDMI core, 0.25 um CMOS standard unit memory. S3C44B0X also provides the following parts: $8 \mathrm{~KB}$ Cache, optional internal SRAM, 2 channels UART, 4 channel DMA, 8 channels of the 12 bit ADC channels, the I2C/I2S bus interface, synchronous SIO interface and pairs of clock PLL, and other resources. [4]

Because it does not have internal FLASH, S3C44B0X puts FLASH Chip MBM29LV160T into the system. According to the intrinsic characteristics of the S3C44B0X, it takes Pin nGCS0 as a FLASH optional port to make FLASH addresses start from 0X00000000.

The system uses HY57V641620 chip as external DRAM of S3C44B0X. According to the intrinsic characteristics of the S3C44B0X, it takes Pin MGCS6 as a DRAM optional port to make DRAM addresses start from 0X0C000000. [5]

\section{Design of Temperature Measurement Module}

In order to measure temperatures in many locations simultaneously, the design should put several pieces of temperature sensors on the serial communication bus. In order to achieve high accuracy of temperature monitoring, the design should choose the digital temperature sensor with 8-bit or more bits resolution. In view of the above reasons, this paper select United States Analog Devices (ADI) single-chip digital temperature sensor AD7416. [6]The sensor contains a band gap temperature sensor and a 10-bit ADC, which can convert analog temperature value to digital signal with quantitative intervals of $0.25^{\circ} \mathrm{C}$.Its On-chip registers can be programmed with high and low temperature limits, and an open drain Over-Temperature Indicator(OTI) output is provided, which becomes active when a programmed limit is exceeded. Furthermore, an I2C-compatible serial interface allows the AD7416 registers to be written to and read back; up to eight AD7416 can be connected to a single bus. Specific connection circuit as shown in Fig. 2

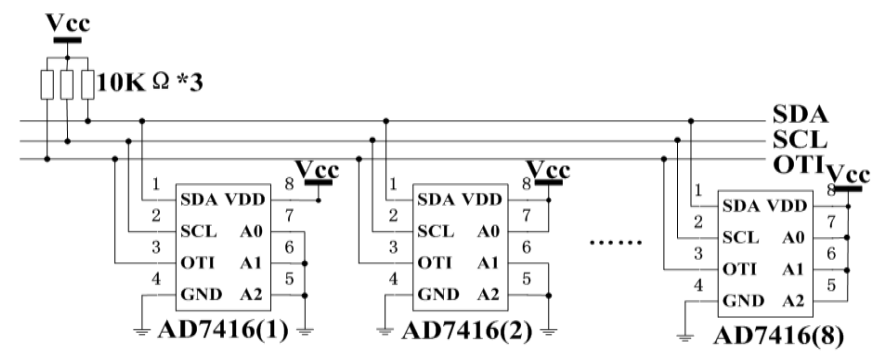

Figure 2. I2C serial bus interface circuit for AD7416

In the interface circuit, the AD7416 carries out the I2C serial bus physical properties by pins SCL and SDA which are connected to pull-up resistor. [7]AD7416 Pin A0, A1, A2 are chip 
selecting address lines, each of which can be set to the high level or low, so it can get eight different kinds of state through the combination. Therefore, selectable serial bus address allows connection of up to eight AD7416 to a single I2C serial bus.

\section{Design of Interface Module}

Interface design mainly includes: design of keys and LED digital tubes interface, then design of interface between the system and the master PC. This paper discusses mainly the latter design.

S3C44B0X chip provides 2 serial UART channels. To get data transmission between the greenhouse temperature monitoring system and a portable computer, this paper uses UART0 as asynchronous serial communication interface, which is connected to the asynchronous serial communication interface of the portable computer.[8]

S3C44B0X chip's UART0 interface uses TTL-level, which is expressed in high and low level voltage state. However, Portable computer asynchronous serial communication interface uses RS-232C level, which is positive or negative voltage to represent logical state. In order to correctly match the electrical characteristics, it must deal with the voltage level and logical relationship between RS-232C and TTL circuits. For this purpose, UART0 serial interface circuit uses MAX3232 chip to deal with the voltage level and logical relationship transformation. The communication hardware connection circuit between system and the master PC serial interface is shown in Fig. 3

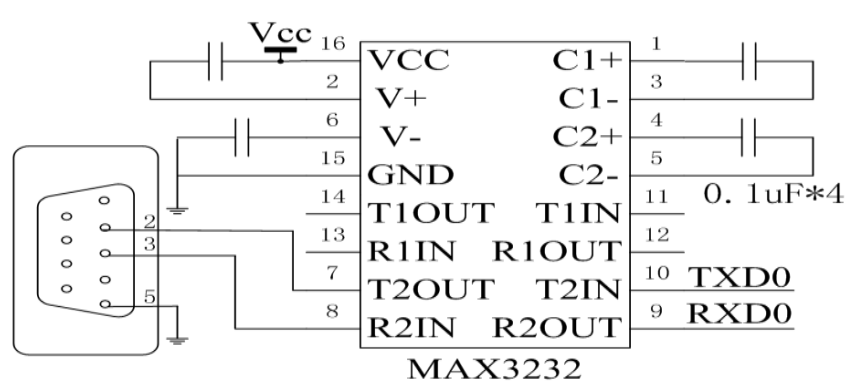

Figure 3. UART0 serial interface circuit

In UART0 serial interface circuit, the data issued by S3C44B0X chip's TXD0 pin is transferred from MAX3232 chip to the portable computer with RS-232C level. At the same time, PC's data is transferred from MAX3232 chip to S3C44B0X chip RXD0 PIN with TTL level.

\section{Porting uCOS-II to ARM Microprocessor}

uCOS- II is the real-time operating system kernel which was first published in 1992. It is used in all kinds of applications such as cameras, medical instruments, ATM machines, industrial robots, and many more. [9]

The uCOS-II includes three parts: The first part is core code, which has nothing to do with the processor. This part includes seven source code files and a header file. The function of the seven source code files are respectively the core management, event management, message queues management, memory management, message management, semaphores management, task scheduling and time management. The second part is configuration code, including two header files which are used to control the number of event control blocks and whether containing news management relevant code, etc. The third part is processor porting section, which includes a header file, an assembly file and a c code file.

\section{Design of Network Communication Module}

The network module uses 10M Ethernet control chip RTL8019. After transplantation of embedded operating system, ARM microprocessors make small TCP/IP protocol stack into the system. Editing 
TCP communication is divided into server or client. Server needs to listen for connection; only after the connection with the client it can deal with the data processing. Client is an active connection to a server; after the connection with the client it can deal with the data processing.[10]

In clients receive task of the network communication, creating a socket, binding Port NO, active to connect to the server, receiving data server code are as follows:

$\mathrm{s}={ }^{*}$ socket $(0,0,0)$;

ei $=$ bind $(($ SOCKET $*) \& s,($ struct sockaddr*)\&cliaddr, sizeof(cliaddr));

while (1)

\{

Temp $=$ connect $\left(\left(\right.\right.$ SOCKET $\left.^{*}\right) \& s,($ struct sockaddr*)\&servaddr,sizeof(servaddr $)$ );

If (Temp! =0xff)

do \{

TempLength=recv( Temp,tempdata ,400, 0$)$;

OSTimeDly(70);

\} while (! TempLength);

\section{Summary}

The application of ARM microprocessor in modern agriculture monitoring field, discussed above in this paper, uses high speed and low power ARM microprocessors instead of ordinary low speed MCS-51 series single-chip for high speed data processing and a number of external interrupt response in real time. It also uses high precision digital temperature sensor to get precise measurement temperature, which improves temperature measurement instrument performance. For this later product development and product upgrade, it may consider providing several kinds of human-computer interfaces between the temperature measurement terminal and the PC.

\section{Acknowledgements}

This paper is supported by Youth Fund Project of Jilin Agricultural Science and Technology University (NO.2015224).

This paper is supported by Key Subject Cultivation Project of Jilin Agricultural Science and Technology University (NO.2015X093).

This paper is supported by Higher Education Scientific Research Project of Jilin Provincial Institute for Higher Education (JGJX2015C90) and supported by Jilin Province education science 12th Five-Year foundation (GH14335).

\section{References}

[1] D.L.Xiong:Crop Growth Remote Sensing Monitoring and its Application, Sensors and Transducers, Vol.169(2014)No.4,p.174.

[2] F G Montoya:A monitoring system for intensive agriculture based on mesh networks and the android system, Computers and Electronics in Agriculture,Vol.99(2013),p.14.

[3] Lutful Karim:Sensor-based M2M Agriculture Monitoring Systems for Developing Countries State and Challenges,Network Protocols and Algorithms,Vol.5(2013)No.3,p.68.

[4] Mauro Agnoletti:Agriculture and land management: the landscape monitoring system in Tuscany,Italian Journal of Agronomy, Vol.4(2011), No.3,p.53.

[5] Young-Duk Kim:On the design of beacon based wireless sensor network for agricultural emergency monitoring systems,Computer Standards \& Interfaces, Vol.36(2014)No.2,p.288. 
[6] Yuan Guai Lin:An Intelligent Monitoring System for Agriculture Based on Zigbee Wireless Sensor Networks,Advanced Materials Research, Vol.1518(2012)No.383,p.4358.

[7] M. Quattrocchi:Evaluation of diagnostic reference levels for angiography system using a radiation dose monitoring system,Physica Medica,Vol.32 (2016)No.1,p.1.

[8] D Gouwanda:Periodical gait asymmetry assessment using real-time wireless gyroscopes gait monitoring system,Journal of Medical Engineering \& Technology,Vol.32(2011)No.1,p.432.

[9] Luca Salvati:The Integrated Assessment of Land Degradation,Italian Journal of Agronomy, Vol.4(2011)No.3,p.77.

[10]Habib F. Rashvand:Distributed Sensor Systems(John Wiley \& Sons, Ltd,UK 2012 ),p.10. 